\title{
TSC1 and TSC2 Gene Mutations in Chinese Tuberous Sclerosis Complex Patients Clinically Characterized by Epilepsy
}

\author{
Jing He, Wenjing Zhou, Jie Shi, Jiuluan Lin, Bingqing Zhang, and Zhaohui Sun
}

Objective: Tuberous sclerosis complex (TSC) is a multisystem disease. Variants in the TSC1 and TSC2 genes have been reported to be associated with TSC and are considered pathogenic. The purpose of this study was to determine the genetic mutations and expression patterns of TSC1 and TSC2 in 21 Chinese patients suffering from TSC who were clinically characterized by epilepsy.

Methods: Peripheral blood samples were taken from 21 patients, their parents, and other family members. Their TSC1 and TSC2 genes were sequenced through next-generation sequencing to identify all variants.

Results: We identified variants in 17/21 patients in either their TSC1 or TSC2 genes: 6 patients had TSC1 mutations and 11 had TSC 2 mutations. There were 13 spontaneous mutations, and 3 that had been inherited from a parent. The mutations were classified by types: there were three missense mutations, five frameshift mutations, two splice site mutations, four nonsense mutations, two single codon deletions resulting the loss of an amino acid, and one large fragment deletion. Six of the mutations have not been previously reported.

Conclusion: The genotypic analysis of Chinese TSC patients who are clinically characterized by epilepsy can potentially be useful for genetic counseling and prenatal diagnoses for patients and their families.

Keywords: tuberous sclerosis complex, epilepsy, TSC1 gene mutation, TSC2 gene mutation

\section{Introduction}

$\mathbf{T}$ UBEROUS SCLEROSIS COMPLEX (TSC) is an autosomal dominant hereditary disease caused by mutations in the TSC1 and TSC2 genes. It is a multisystem disorder involving the heart, kidneys, lungs, and central nervous system, resulting in intellectual disability, seizures, and autism (Caban et al., 2016). Recent studies estimate a frequency of $1 / 6000$ to $1 /$ 10,000 live births (Hong et al., 2016). Both TSC1 and TSC2 are tumor suppressor genes. The TSC1 gene locus is located in the 9q34 region encoding hamartin, and the TSC2 gene locus is located in the $16 \mathrm{p} 13.3$ region encoding tuberin (Yates et al., 2011). Both proteins are widely expressed in tissues, and form a hamartin-tuberin complex in vivo. If mutations occur in either of these genes, the function of the hamartin-tuberin dimer will be affected; and the signal transduction pathway of the mammalian target of rapamycin (mTOR) complex 1 will be abnormally activated, resulting in the development of TSC (Van Slegtenhorst et al., 1998; Li et al., 2004; Henske et al., 2016).

\section{Methods}

Patient demographic data

We enrolled 21 (11 males and 10 females) TSC patients in this study who were clinically characterized by epilepsy and hospitalized in our department from 2016 to 2018. All patients had been treated with $>3$ drugs for a period of $>10$ months, and all of them were reported to suffer from epilepsy and were refractory to treatment.

\section{Gene detection methods}

The DNA isolated from peripheral blood leukocytes was fragmented, and libraries were prepared according to the recommended protocols of Illumina; the library DNA was captured using an Agilent SSELXT Human All Exon V6 kit. Enriched capture libraries were sequenced on a HiSeq X-ten or HiSeq 2500 platform (Illumina) using 150 base paired-end sequencing. The average sequencing depth of the target regions was $\times 100$, and the coverage of the target exons was $99.8 \%$.

Data analysis consisted of filtering out the Illumina sequencing adapters and low-quality reads $(<80 \mathrm{bp})$ using fastq_mcf. Postquality control, the clean reads were mapped to the UCSC GRCh38/hg38 human reference genome using BWA (Burrows Wheeler Aligner). Duplicated reads were removed using Picard Tools, and only unique mapping reads were used for variation detection. Next, single nucleotide polymorphisms (SNPs) and indel variants were detected

Department of Epilepsy Center, Tsinghua University Yuquan Hospital, Beijing, China. 

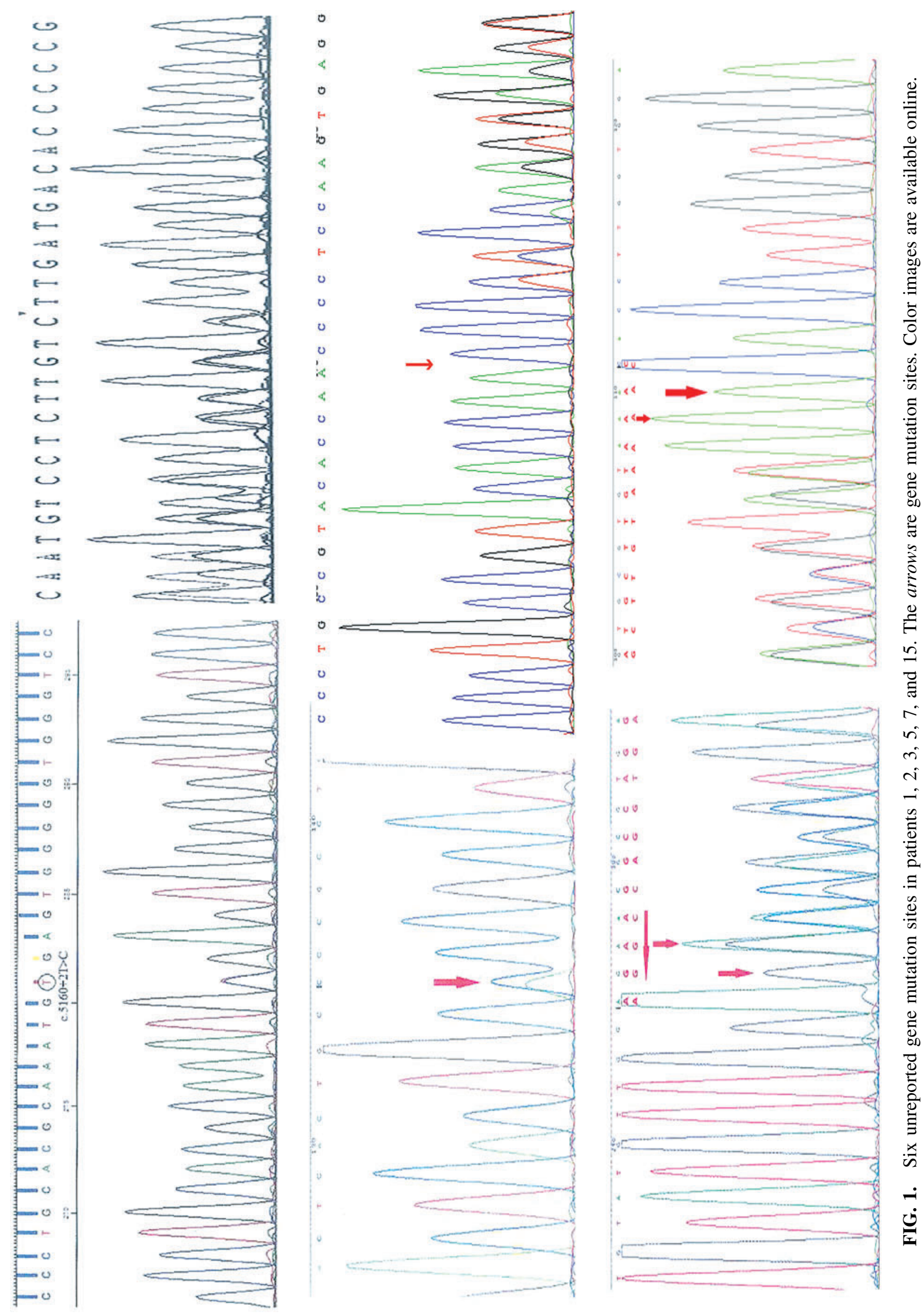
Table 1. Gene Mutation Data

\begin{tabular}{|c|c|c|c|c|c|c|c|c|}
\hline Patient & $\begin{array}{l}\text { Mutant } \\
\text { gene }\end{array}$ & $\begin{array}{l}\text { Mutation } \\
\text { type }\end{array}$ & Exon & Base alteration & $\begin{array}{l}\text { Amino acid } \\
\text { alteration }\end{array}$ & $\begin{array}{l}\text { Reported } \\
\text { or not? }\end{array}$ & Source & Pathogenicity \\
\hline 1 & TSC2 & Splice site & Intron 40 & c. $5160+2 \mathrm{~T}>\mathrm{C}$ & No alteration & No & Novel & Pathogenicity \\
\hline 2 & $T S C 2$ & Nonsense & Exon25 & c.2771_2772del & p.Phe924* & No & Unknown & Pathogenicity \\
\hline 3 & TSC2 & Missense & Exon 18 & c. $1880 \overline{\mathrm{A}}>\mathrm{C}$ & p.His627Pro & No & Mother & Pathogenicity \\
\hline 4 & $T S C 2$ & Missense & Exon24 & c. $2713 \mathrm{C}>\mathrm{T}$ & p.Arg905Trp & Yes & Novel & Pathogenicity \\
\hline 5 & TSC2 & Frameshift & Exon 23 & c. $2634 \mathrm{del}$ & p.Ser879Profs $* 15$ & No & Novel & Pathogenicity \\
\hline 6 & TSC2 & $\begin{array}{l}\text { Amino acid } \\
\text { deletion }\end{array}$ & Exon38 & c.4912_4914del & p.Lys1638del & Yes & Novel & Pathogenicity \\
\hline 7 & TSC2 & $\begin{array}{c}\text { Amino acid } \\
\text { deletion }\end{array}$ & Exon34 & c.4452_4454del & p.Thr1485del & No & Father & Pathogenicity \\
\hline 8 & $T S C 2$ & Missense & Exon27 & c. $3095 \mathrm{G}>\mathrm{C}$ & p.Arg1032Pro & Yes & Novel & Pathogenicity \\
\hline 9 & $T S C 2$ & Frameshift & Exon34 & c. $4258 \_4261 \mathrm{del}$ & p.Ser1420Glyfs $* 55$ & Yes & Novel & Pathogenicity \\
\hline 10 & $T S C 2$ & $\begin{array}{l}\text { Large } \\
\text { fragment } \\
\text { deletion }\end{array}$ & Exon10-16 & Exon10-16 del & exon $10-16$ del & Yes & Novel & Pathogenicity \\
\hline 11 & $T S C 2$ & Nonsense & Exon8 & c. $684 \mathrm{C}>\mathrm{A}$ & p.Tyr228* & Yes & Novel & Pathogenicity \\
\hline 12 & TSC1 & Splice site & Intron9 & c. $913+1 \mathrm{G}>\mathrm{A}$ & No alteration & Yes & Novel & Pathogenicity \\
\hline 13 & TSC1 & Nonsense & Exon21 & c. $2692 \mathrm{C}>\mathrm{T}$ & p.Gln898* & Yes & Novel & Pathogenicity \\
\hline 14 & TSC1 & Frameshift & Exon 18 & c.2356_2359del & p.Glu787Asnfs*19 & Yes & Novel & Pathogenicity \\
\hline 15 & TSC1 & Frameshift & Exon4 & c.127_128dup & p.Asn43Lysfs*20 & No & Novel & Pathogenicity \\
\hline 16 & TSC1 & Frameshift & Exon 10 & c.989dup & p.Ser331Glufs*10 & Yes & Novel & Pathogenicity \\
\hline 17 & TSC1 & Nonsense & Exon 17 & c. $2074 \mathrm{C}>\mathrm{T}$ & p.Arg692* & Yes & Mother & Pathogenicity \\
\hline 18 & & & Not found & & & & & \\
\hline 19 & & & Not found & & & & & \\
\hline 20 & & & Not found & & & & & \\
\hline 21 & & & Not found & & & & & \\
\hline
\end{tabular}

TSC, tuberous sclerosis complex.

with the GATK HaplotypeCaller. Then, the GATK Variant Filtration tool was utilized to filter the variants. The filtering standards were as follows: (1) variants with mapping qualities <30; (2) the total mapping quality zero reads $<4$; (3) approximate read depth $<5$; (4) QUAL <50.0; (5) phredscaled $p$-value using Fisher's exact test to detect strand bias $>10.0$. After these two steps, the data were transformed to a variant call format, and variants were further annotated by ANNOVAR; in addition, they were run against multiple databases, including the 1000 genomes project, ESP6500, dbSNP, EXAC, and HGMD. Variant functions were predicted by SIFT, PolyPhen-2, MutationTaster, and GERP++. Of all the mutations, we focused on mutations that ACMG (American College of Medical Genetics) identified as pathogenic, likely pathogenic, and variant of undetermined significance.

\section{Results}

The TSC gene was sequenced for all 21 patients enrolled in the study, and mutations in one or the other of the TSC genes were found in 17 patients. Six subjects had TSC1 gene mutations, which accounts for $28.6 \%, 11$ subjects had TSC2 gene mutations, which accounts for $52.4 \%$, and 4 patients had no TSC gene mutations identified accounting for $19.0 \%$. Among all of the variants detected, 13 were spontaneous $(76.5 \%), 3$ patients inherited their mutations from 1 of their parents, and 1 patient had an unknown source as no blood samples were taken from his parents. The TSC2 gene mutations in eight of the patients were spontaneous, two were inherited, and one was undeter- mined as to origin. The TSCl gene mutations in five of the patients were spontaneous, and one was inherited from a parent.

In this study, we found six novel mutation sites (Fig. 1) accounting for $35.3 \%$ of all TSC mutations characterized in this study, among which five were in the TSC2 gene: c.5160+

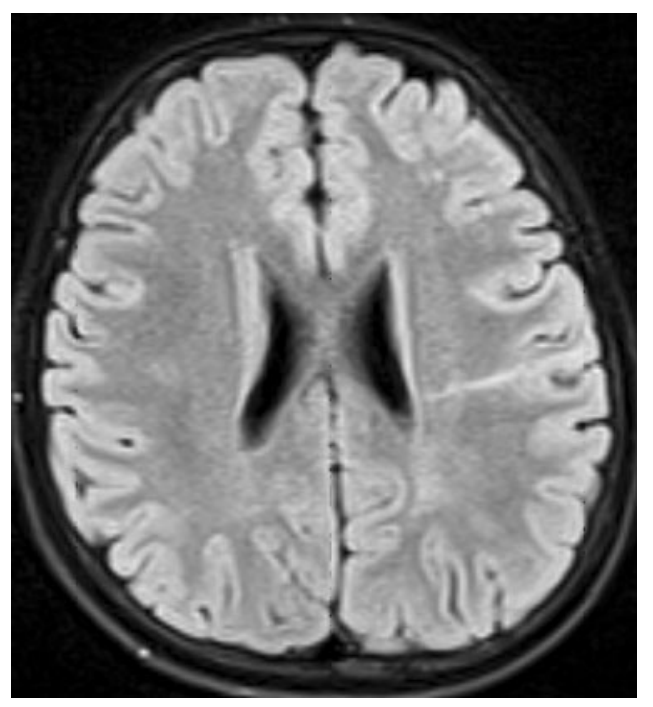

FIG. 2. MRI manifestation. MRI showing manifestations in patient 3 with high signal in left central tectum on T2 flair. MRI, magnetic resonance imaging. 
2T $>$ C, no amino acid alteration; c.2771_2772del, p.Phe924*; c.1880A >C, p.His627Pro; c.2634del, p.Ser879Profs*15; c.4452_4454del, p.Thr1485del; and one in the TSC1 gene: c.127_128dup, p.Asn43Lysfs*20.

The likely pathogenic variants included five frameshift mutations $(29.4 \%)$, three missense mutations $(17.6 \%)$, four nonsense mutations $(23.5 \%)$, two splice site mutations (11.8\%), two codon (single amino acid) deletions (11.8\%), and one large fragment deletion of multiple exons (5.9\%) (Table 1).

DNA sequencing of the causative mutations for the probands resulted in improved diagnoses and genetic counseling for the patients' families in some cases. Patient 3 had a family history of TSC. The patient's brother had a history of epilepsy, for which he received antiepileptic treatment, but had not been diagnosed with TSC despite the fact that he had white spots and a coffee spot on his skin (Fig. 2). Blood samples from other family members showed that the proband's grandfather, mother, and niece had the same mutation in their TSC2 gene; interestingly, none of them suffered from epilepsy.

\section{Discussion}

Epilepsy is a significant cause of morbidity and mortality (Jeong and Wong, 2016), and one of the most common neurological symptoms in patients with TSC, with a reported prevalence from $62 \%$ to $93 \%$ (Krueger, 2013). The gain of function associated with mutations in the TSC1/ TSC2 proteins, which are directly involved in mTOR regulation, affects many functions during development and maturation, including cell growth and proliferation, energy metabolism, inflammation, and synaptic plasticity (Yasin et al., 2013). Such a hyperactivation of the mTOR pathway generates dysmorphic neurons that are as "epileptic generators" in TSC (Kannan et al., 2016). Whether the mutations we reported are more likely to cause refractory epilepsy is uncertain.

Spontaneous mutations in the TSC genes were responsible for $76.5 \%$ of the TSC cases in this study; this is similar to previous reports (Sancak et al., 2005). Therefore, we conclude that spontaneous mutations are the principal driver of new cases. Among the TSC patients who had no reported familial history, a genetic analysis can aid in making definite diagnoses both pre- and antenatally (Yates, 2006; Schwartz et al., 2007).

Previous studies have reported that the average rate of TSC patients with no TSC gene mutations was $14.4 \%$ (Nabbout et al., 2018); our findings of $19 \%$ are consistent with this. Among all gene mutations, frameshift mutations were the most common type in our study, accounting for $29.4 \%$.

It was reported that TSC2 gene mutations are more common than TSC1 gene mutations (Peron et al., 2018a, $2018 \mathrm{~b}$ ) and that they are associated with an increased risk of seizures (Dabora et al., 2001). The fact that our patients had intractable epilepsy may partially account for our finding more TSC2 gene mutations than TSC1 gene mutations in our cohort.

This study is not without its limitations. First, the protein changes predicted to be encoded by the gene and the expression of individual mutant genes were not analyzed. In future studies we plan to further study protein function. In addition, this study had a relatively small sample size and, therefore, cannot fully represent the characteristics of TSC among the Chinese people.

\section{Conclusion}

In this study, the majority of the mutations identified in the TSC genes were spontaneous. For suspected TSC patients, gene detection can be performed before epileptic seizures occur to achieve an early and definite diagnosis. This study provides important data for family-based diagnoses and genetic counseling for TSC.

\section{Acknowledgments}

The authors thank all the participants.

\section{Author Disclosure Statement}

No competing financial interests exist.

\section{Funding Information}

No funding was received for this article.

\section{References}

Caban C, Khan N, Hasbani DM, et al. (2016) Genetics of tuberous sclerosis complex: implications for clinical practice. Appl Clin Genet 10:1-8.

Dabora SL, Jozwiak S, Franz DN, et al. (2001) Mutational analysis in a cohort of 224 tuberous sclerosis patients indicates increased severity of TSC2, compared with TSC1, disease in multiple organs. Am J Hum Genet 68:64-80.

Henske EP, Jóźwiak S, Kingswood JC, et al. (2016) Tuberous sclerosis complex. Nat Rev Dis Primers 2:16035.

Hong CH, Tu HP, Lin JR, et al. (2016) An estimation of the incidence of tuberous sclerosis complex in a nationwide retrospective cohort study (1997-2010). Br J Dermatol 174: 1282-1289.

Jeong A, Wong M (2016) Systemic disease manifestations associated with epilepsy in tuberous sclerosis complex. Epilepsia 57:1443-1449.

Kannan L, Vogrin S, Bailey C, et al. (2016) Centre of epileptogenic tubers generate and propagate seizures in tuberous sclerosis. Brain 139:2653-2667.

Krueger DA (2013) Management of CNS-related disease manifestations in patients with tuberous sclerosis complex. Curr Treat Options Neurol 15:618-633.

Li Y, Corradetti MN, Inoki K, et al. (2004) TSC2: filling the GAP in the mTOR signaling pathway. Trends Biochem Sci 29:32-38.

Nabbout R, Belousova E, Benedik MP, et al. (2018) Epilepsy in tuberous sclerosis complex: findings from the TOSCA Study. Epilepsia Open 4:73-84.

Peron A, Au KS, Northrup H (2018a) Genetics, genomics, and genotype-phenotype correlations of TSC: insights for clinical practice. Am J Med Genet C Semin Med Genet 178: 281-290.

Peron A, Vignoli A, La Briola F, et al. (2018b) Deep phenotyping of patients with tuberous sclerosis complex and no mutation identified in TSC1 and TSC2. Eur J Med Genet 61: 403-410.

Sancak O, Nellist M, Goedbloed M, et al.(2005) Mutational analysis of the TSC 1 and TSC2 genes in a diagnostic setting: 
genotype-phenotype correlations and comparison of diagnostic DNA techniques in tuberous sclerosis complex. Eur $\mathbf{J}$ Hum Genet 13:731-741.

Schwartz RA, Fernandez G, Kotulska K, et al. (2007) Tuberous sclerosis complex: advances in diagnosis, genetics, and management. J Am Acad Dermatol 57:189-202.

Van Slegtenhorst M, Nellist M, Nagelkerken B, et al. (1998) Interaction between hamartin and tuberin, the TSC1 and TSC2 gene products. Hum Mol Genet 7:1053-1057.

Yasin SA, Ali AM, Tata M, et al. (2013) mTOR-dependent abnormalities in autophagy characterize human malformations of cortical development: evidence from focal cortical dysplasia and tuberous sclerosis. Acta Neuropathol 126:207-218.

Yates JR (2006) Tuberous sclerosis. Eur J Hum Genet 14: 1065-1073.
Yates JR, Maclean C, Higgins JN, et al. (2011) The Tuberous Sclerosis 2000 Study: presentation, initial assessments and implications for diagnosis and management. Arch Dis Child 96:1020-1025.

Address correspondence to: Wenjing Zhou, MD Department of Epilepsy Center Tsinghua University Yuquan Hospital

No. 5 Shijingshan Road Shijingshan District Beijing 100040

China

E-mail: closejeo@hotmail.com 